\title{
Innovating Through Measurement
}

\author{
Bruce I. Reiner
}

Published online: 4 December 2012

(C) Society for Imaging Informatics in Medicine 2012

"If you want to effect real and substantive change, measure it."

You cannot go anywhere or read anything today without being bombarded with innovation strategies, which have become the mantra for all business pundits and selfproclaimed entrepreneurs. While these innovation strategies are designed to transform business and profitability, the reality is that innovation is frequently talked about but rarely experienced. The medical imaging and informatics communities are especially prone to a vast array of innovation and marketing hype with little substantive change to show for the efforts. I have found amusement at attending RSNA meetings where large imaging and informatics vendors with vast resources display their futuristic innovation visions which never reach the marketplace. Their marketing ploys look and sound great, but if they never become commercialized what is their purpose in the first place?

The consolidation and maturation of medical imaging informatics has led to a number of disconcerting effects within everyday practice; perhaps the most important of which is a dearth of technology innovation. Why would one expect a large risk averse company with an established market share and installation base to parasitize itself and create new technology, only to undermine its existing product line? The technology consumer would answer that true innovation will expand the purview of the company and help differentiate it from its competitors who lack true innovative vision (think Apple). But the financial experts within the companies often think differently and prefer a conservative approach where current technology is continuously promoted through creative bundling, incremental improvements, and

B. I. Reiner $(\bowtie)$

Department of Radiology, Veterans Affairs Maryland Healthcare

System, 10 North Greene Street,

Baltimore, MD 21201, USA

e-mail: breiner1@comcast.net scare tactics (often related to the impending demise of the competition).

If the community of service providers cannot depend on traditional technology producers to innovate, what is the logical alternative? The current economic pressures and commoditization trends have continuously reduced reimbursements (both technical and professional), which create increasing pressures to increase productivity and workflow in order to maintain revenue. Instead of thinking about innovation, the medical imaging community is largely resigned to forging ahead and "making do" with existing practice strategies and technologies. Simply stated, innovation demands precious resources and optimism which are currently lacking. Perhaps we can innovate another day; when the sun is shining, cash is readily available, and creativity is financially rewarded.

Perhaps the answer to this innovation vacuum is right in front of us, so plain to see that we ignore it. While we look to technology producers to drive innovation, we often minimize our own innovative abilities. While we, the collective community of medical imaging service providers, may not know how to create technology, what we do know is how to enhance quality and safety. We can define quality deliverables in a number of ways including technical imaging quality, radiation safety, diagnostic accuracy, and improved timeliness and operational efficiency. In the end, the common denominator is clinical outcomes, which is (or should be) the holy grail of all medical innovation efforts.

So if we want to create innovation strategies which positively affect any or all of these quality deliverables, we need to determine where and how to begin. In my opinion, the most logical starting place is the data which we create, store, and analyze in everyday practice. Numerical data abounds and can take the form of operational efficiency measures (e.g., report turnaround times), diagnostic accuracy (e.g., MQSA positive and negative predictive values), and patient safety (e.g., radiation exposure measures). While imaging data is ubiquitous in everyday practice, there has been little effort made to date to standardize its analysis. This can be 
done in a variety of ways; simplistically beginning with subjective image quality analysis at the point of care to the more advanced and objective analysis of technical image quality using standardized computer algorithms (1). The ultimate goal is to create standardized methods for recording data at all steps of the medical imaging cycle; while individually and collectively analyzing the impact of each participant and technology on clinical outcomes (2-5). By doing so, we could begin to shed critical light on best practice guidelines, interaction effects, and comparative technology performance. Rather than view the derived data analytics as potentially punitive; the community of end-users should instead view this as a tool for empowerment and enhanced education. Individual and institutional providers can begin to understand where their relative strengths and deficiencies lie; and utilize the data for targeted education and training, workflow modification, technology deployment, and decision support.

Unfortunately, this concept of "quantitative accountability" is not universally embraced by the medical imaging community, largely out of fear of exposing quality and safety deficiencies on the part of some service providers. Many providers fear that widespread use and public access of this data can have a detrimental economic impact on their practice. While this concern is certainly legitimate, it does not address the central premise that all medical imaging stakeholders (including consumers) should have the ability to make educated and informed decisions related to employment, provider selection, technology procurement, research, and education / training. This can only be realistically accomplished through analysis of objective, reproducible, and standardized data. Instead of trying to maintain artificial edifices preventing data access, stakeholders should in my opinion devote their energies to ensuring that the methodology and infrastructure used for quantitative accountability is accurate, secure, and accessible. The hope is that this data will ultimately serve as a source of empowerment, selfimprovement, and enhanced economic security; especially for those providers who can consistently demonstrate high levels of quality and safety in their practice. Those providers demonstrating lower performance analytics will be provided with a fair and objective means of self-improvement, which if used efficiently can lead to improved clinical and economic outcomes. In the end, market economics will determine the inherent value of these service deliverables, with selection of service providers based upon objective quality and safety data; as opposed to the current practice which sometimes employ nefarious tactics such as self-referral, favoritism, kickbacks, and predetermined contractual obligations (6). If and when such a system is employed, the transparency of the data will in theory become a driver in innovation. Service providers will seek out and create new strategies which provide them with a competitive edge, which will ultimately create a practice environment predicated on improved quality and safety.

The technology vendor community can also benefit from this standardized (i.e., non-proprietary) data-driven analysis in a number of ways. They would not only learn the relative strengths and deficiencies of their products, but also discover innovation opportunities to help guide future R \& D efforts. By using the same data to show beneficial "cause and effect" relationships between technology innovation and improved clinical outcomes, marketing efforts will be objectively validated and consumers will become empowered. This could actually expand the market and favor those technology vendors and service providers who can strategically create and adopt innovation in accordance with improved clinical outcomes. The hope is that this newfound data-derived knowledge will drive innovation in technology creation, service delivery, and market economics. While simplistic in nature, the best way to effect change and innovation is through measurement. Once we all accept the fact that the current innovation model is inherently flawed and often fosters inertia, we can begin to move forward. If we create objective and reproducible methods for analyzing quality and safety data in medical imaging, we can begin to create quantitative accountability on a truly level playing field. Those participants who choose not to participate run the risk of becoming marginalized and economically non-viable. The collective medical imaging community has the ability to control its own destiny and drive future innovation through prospective and longitudinal data analysis. The sooner we begin, the sooner we will benefit.

This compelling need for data-driven innovation will be highlighted in a new innovation series in the Journal of Digital Imaging $(J D I)$. The planned topics will vary in focus but have the common themes of data standardization, adaptation to end-user needs and attributes, and a shared focus on quality and workflow improvement. I would invite the readership to actively participate in the process by identifying specific areas in need of substantive change, feedback on the proposed concepts, and new innovation ideas. This feedback can be provided through traditional letters to the editor or via the JDI website, which is introducing an innovation forum. Vendor participation is equally encouraged and should provide a practical balance to the collective experience of the administrative, IT, and clinical communities. The goal is to serve as 
catalysts for collaborative change based upon the collective thoughts, needs, and experience of all stakeholders.

\section{References}

1. Reiner BI: Automating quality assurance for digital radiography. J Am Coll Radiol 7:486-490, 2009

2. Reiner BI: Quantifying radiation safety and quality in medical imaging. Part I: creating the infrastructure. J Am Coll Radiol 8:558-561, 2009
3. Reiner BI: Quantifying radiation safety and quality in medical imaging. Part II: the radiation scorecard. J Am Coll Radiol 9:615619, 2009

4. Reiner BI: Quantifying radiation safety and quality in medical imaging. Part III: the quality assurance scorecard. J Am Coll Radiol 10:694-700, 2009

5. Reiner BI: Quantifying radiation safety and quality in medical imaging. Part IV: the medical imaging agent scorecard. J Am Coll Radiol 2:120-124, 2010

6. Reiner BI: Transforming healthcare service delivery and provider selection. J Digit Imaging 3:373-377, 2011 